\title{
Correlation Analysis of Sleep Quality and Youth Ischemic Stroke
}

\author{
Shunqing Zhang, ${ }^{1,2}$ Cheng Chang, ${ }^{3}$ Juan Zhang, ${ }^{4}$ Bo Song, ${ }^{1}$ Hui Fang, ${ }^{1}$ and YuMing Xu \\ ${ }^{1}$ Neurological Department of the First Affiliated Hospital of Zhengzhou University, Zhengzhou 450052, China \\ ${ }^{2}$ Neurological Department of Puyang People's Hospital, Puyang 457000, China \\ ${ }^{3}$ Human Anatomy Department of Zhengzhou University, Zhengzhou 450001, China \\ ${ }^{4}$ Nuclear Medicine Department of the Fifth Clinical Medicine Hospital of Beijing University, Beijing 100730, China
}

Correspondence should be addressed to YuMing Xu; yuming.xu@sohu.com

Received 3 April 2014; Revised 7 July 2014; Accepted 17 July 2014; Published 5 August 2014

Academic Editor: Di Lazzaro Vincenzo

Copyright ( 2014 Shunqing Zhang et al. This is an open access article distributed under the Creative Commons Attribution License, which permits unrestricted use, distribution, and reproduction in any medium, provided the original work is properly cited.

\begin{abstract}
Objective. To study risk factors related to ischemic stroke (IS) in youth and the influence of sleep quality on youth ischemic stroke incidence. Methods. 223 patients aged 18 to 45 years who were admitted to Puyang People's Hospital from June 2011 to February 2013 with a first-ever ischemic stroke were selected as the research cases. 158 young people with a normal physical examination were selected as the control group. The Pittsburgh Sleep Quality Index (PSQI) questionnaire was used to analyse the correlation between sleep quality and youth IS incidence. The US National Institutes of Health Stroke Scale (NIHSS) and modified Rankin Scale (MRS) scores were used to assess cases' state of illness and prognosis three months after IS. Results. Univariate and multivariate logistic regression analysis showed that the association of these risk factors with youth IS incidence, from highest to lowest, was hypertension, hyperlipidaemia, smoking history, high homocysteine, the quality of sleep, family history of stroke, and alcoholism. Poor sleep quality ranked fifth among all risk factors and was positively correlated with poor prognosis for youth IS patients. Conclusion. The results of this study showed that sleep quality is an important factor in the pathogenesis and prognosis of youth IS.
\end{abstract}

\section{Introduction}

Ischemic stroke (IS) has become a high incidence, high morbidity, and high mortality disease in recent years, and its incidence is increasing significantly in young people [1]. Youth IS is defined as patients who are 18 to 45 years old with ischemic stroke, and it accounts for approximately 5 to $10 \%$ of all IS cases $[2,3]$. Youth IS not only affects the patient's quality of life and work but also inflicts a heavy economic burden on families and society. Therefore, exploring IS risk factors and proactive prevention strategies are important. Youth IS risk factors and active prevention research on youth IS have attracted the attention of medical workers; however, risk factors reported in China are inconsistent with that in other countries [4-6]. Studies have shown that sleep quality has a close relationship with ischemic stroke incidence and prognosis [7]. Current research mainly focuses on the mechanisms of sleep disorders after cerebral infarction in youth IS patients. Few studies have explored the role of sleep quality in youth IS pathogenesis or investigated whether sleep quality is a cause of youth IS incidence and prognosis $[8,9]$. This study extracted data on 223 youth IS patients from Puyang City People's Hospital to observe and analyse the relationship between sleep quality and youth IS, find new youth IS risk and prognostic factors, and provide clinical evidence for the prevention and treatment of youth IS.

\section{Materials and Methods}

\subsection{Research Materials}

2.1.1. Selection of Study Subjects. In total, 223 patients aged 18 to 45 years who were admitted to Puyang People's Hospital from June 2011 to February 2013 with a first-ever ischemic stroke were selected as the research cases. Young people without obvious discomfort and symptoms (aged 18 to 45 years) were elected as the control group by normal physical examination in the same hospital. The study was approved by the Ethics Committee of Puyang City People's Hospital. 
2.1.2. Inclusion and Exclusion Criteria. Patient inclusion criteria were as follows: (1) fulfilment of 2007 AHS/ASA ischemic stroke clinical diagnostic criteria [10]; (2) hospitalization within $72 \mathrm{~h}$ after IS onset; (3) clear focal neurological deficit symptoms and signs lasting more than $24 \mathrm{~h}$ without relief; (4) head MRI diffusion sequences confirming a clear ischemic infarct; and (5) consciousness. Patient exclusion criteria were as follows: (1) cerebral haemorrhage by confirming head CT; (2) thrombolysis within the time window; (3) severe heart, liver, or kidney disease and/or cancer; (4) severe mental illness; (5) ischemic stroke history or sequela; (6) impairment in muscle strength and the ability to make informed life decisions, including suffering from orthopaedic, rheumatic, and/or mental disorders; and (7) failure to consent to followup or participation in the establishment of a database.

\subsection{Research Methods}

2.2.1. Data Collection and Outcome Measures. Patients included in the observation group were hospitalized within $72 \mathrm{~h}$ after IS onset. Once hospitalized, details regarding patients' past medical, personal, and family histories were obtained and recorded. In addition, IS-related data (hypertension, hyperlipidaemia, smoking history, alcohol consumption history, sleep quality, high homocysteine, diabetes, heart disease, cerebrovascular disease, and family history) was collected from medical records. On the second day of hospitalization, patient improvement was monitored via vital signs, ECG, blood biochemistry tests, carotid ultrasound, the Pittsburgh Sleep Quality Index (PSQI) questionnaire score, the US National Institutes of Health Stroke Scale (NIHSS) score, and the modified Rankin Scale (MRS) score. Patients with mild sleep disorders were made nondrug spiritual comfort treatment. For that with severe sleep disorders were made shortterm medication within hospitalization. For severe sleep disorder cases, PSQI scores were recorded as $\geq 7$ (poor sleep quality). Admission time, treatment options, and treatment process for all cases were found to have no significant differences by Power-Test analysis. This ensured that the data collected from different cases were comparable.

A detailed explanation about youth IS pathogenesis and harm and common risk factors (hypertension, high cholesterol, smoking, alcoholism, sleep quality, high homocysteine, and diabetes) was provided to observation group members for health knowledge and education. Patients in the observation group were required to have the follow-up examination in the morning, with fasting, three months $(90 \pm 3 \mathrm{~d})$ after discharge. Additionally, patients were encouraged by attending doctors to quit smoking, limit alcohol, control their diet, take medication regularly, monitor their blood pressure and biochemistry (lipids, glucose, homocysteine, and other targets), and record PSQI, NIHSS, and MRS scores during the period of follow-up examination ( $90 \pm 3 \mathrm{~d}$ after discharge). A complete personal database file was established for all patients. The person responsible for data collection, data entry, and follow-up and management and quality control of the database conducted follow-up or home visits.
2.2.2. Imaging Examination. All patients were admitted to the hospital after completion of cranial CT (computerized tomography), MRI (magnetic resonance imaging), neck vascular ultrasound, TCD (transcranial Doppler), MRA (magnetic resonance angiography), and other tests. Some patients also underwent a CTA (CT angiography) or DSA (digital subtraction angiography) examination.

\subsection{Definitions of Risk Factors}

(1) Hypertension [11]: systolic blood pressure $\geq 140$ $\mathrm{mm} \mathrm{Hg}$ and/or diastolic blood pressure $\geq 90 \mathrm{~mm} \mathrm{Hg}$ with a history of taking antihypertensive drugs.

(2) Hyperlipidaemia [12]: total cholesterol (TCH) $\geq$ $5.70 \mathrm{mmol} / \mathrm{L}$ and/or triglyceride $(\mathrm{TG}) \geq 2.04 \mathrm{mmol} / \mathrm{L}$, high-density lipoprotein cholesterol (HDL-C) $<1.0$ $\mathrm{mmol} / \mathrm{L}$, low-density lipoprotein cholesterol (LDLC) $>3.2 \mathrm{mmol} / \mathrm{L}$, lipoprotein(a) $[\mathrm{LP}(\mathrm{a})]>300 \mathrm{mg} / \mathrm{L}$, apolipoprotein $\mathrm{Al}$ (Apo-A1) > 1.09/L, and lipoprotein B100 (Apo-B100) > 1.09/L.

(3) High homocysteine (Hcy) [13]: plasma Hcy levels of 6 to $15 \mu \mathrm{mol} / \mathrm{L}$ (men $<60$ years), 3 to $12 \mu \mathrm{mol} / \mathrm{L}$ (women $<60$ years), or 15 to $20 \mu \mathrm{mol} / \mathrm{L}$ (men and women $>60$ years).

(4) Diabetes [14]: a history of diabetes, two fasting blood sugar readings of $\geq 7.0 \mathrm{mmol} / \mathrm{L}$ or two $2 \mathrm{~h}$ postprandial readings of $\geq 11.1 \mathrm{mmol} / \mathrm{L}$ after IS onset.

(5) Smoking history: $>10$ cigarettes/d for $>12$ months or $>20$ cigarettes/d for $>10$ years even quitting $<10$ years.

(6) Drinking history: $>750 \mathrm{~mL} / \mathrm{d}$ of beer, $>10 \mathrm{~mL} / \mathrm{d}$ of hard alcohol, or $>360 \mathrm{~mL} / \mathrm{d}$ of wine, in accordance with the World Health Organization (WHO) classification criteria.

(7) Family history: history of cerebrovascular disease in the immediate family, such as parents, brothers, and sisters.

(8) Sleep quality: quality of sleep over a one-month period as measured by the Pittsburgh Sleep Quality Index (PSQI). The PSQI is composed of 18 items that assess seven areas of sleep (sleep quality, time to fall asleep, sleep duration, sleep efficiency, sleep disorders, sleep medication, and daytime dysfunction). Each area is given a score of 0 to 3 points, yielding a cumulative PSQI score of 0 to 21 . Cumulative PSQI scores of $<7$ points indicated good sleep, while PSQI scores of $\geq 7$ indicated poor sleep [15].

(9) Exercise: aerobic exercise at least twice per week for more than 30 min each time.

2.4. Prognosis and Outcome Assessment. Results of the modified Rankin Scale outcome assessment were divided into the following categories: MRS score $<3$ (self-care), MRS score $\geq$ 3 (dependent on others), and MRS score $=6$ (death) [16].

2.5. Statistical Analysis. SPSS10.0 was used for statistical analysis. Normally distributed variables were presented as the 
TABLE 1: Risk factors analysis for observation group and youth control group. By univariate analysis, smoking history, drinking history, sleep quality, hypertension, diabetes, hyperlipidaemia, family history of stroke, and high homocysteine were statistically different between youth IS patients and youth control cases $(P<0.05)$.

\begin{tabular}{|c|c|c|c|c|}
\hline Risk factors & Observation group $(n=223)$ & Young control group $(n=158)$ & Statistic & $P$ \\
\hline Age (years) & $38.26 \pm 6.351$ & $35.99 \pm 6.660$ & 1.856 & 0.174 \\
\hline Gender (male) & $170(76.2)$ & $111(70.3)$ & 1.708 & 0.191 \\
\hline Exercise & $21(9.4)$ & $23(14.6)$ & 2.392 & 0.122 \\
\hline Smoking history & $97(43.5)$ & $36(22.8)$ & 17.461 & 0.000 \\
\hline Drinking history & $111(49.8)$ & $52(32.9)$ & 10.744 & 0.001 \\
\hline Sleep quality & $105(47.1)$ & $46(29.1)$ & 12.484 & 0.000 \\
\hline Hypertension & $104(46.6)$ & $30(19.0)$ & 31.007 & 0.000 \\
\hline Diabetes & $44(19.7)$ & $18(11.4)$ & 4.719 & 0.030 \\
\hline Hyperlipidaemia & $74(33.2)$ & $33(20.9)$ & 6.925 & 0.009 \\
\hline Family history of stroke & $79(35.4)$ & $33(20.9)$ & 9.420 & 0.002 \\
\hline High homocysteine & $92(41.3)$ & $34(21.5)$ & 16.275 & 0.000 \\
\hline
\end{tabular}

mean values (standard deviation), nonnormally distributed variables were presented as median values $\left(P_{25}, P_{75}\right)$, and count data were presented as the number of cases (\%). Univariate analysis was performed to examine descriptive statistics and to conduct $x^{2}$ tests for normally distributed continuous variables, $t$-tests, and nonparametric Mann-Whitney tests performed on the level of the variable. Compared variables of differences between observation and control group were screened by univariate analysis. Finally, a multivariate logistic regression was conducted within filtered compared variables (risk factors) to identify statistically significant variables associated with youth IS. $P$ values of $<0.05$ were considered statistically significant.

\section{Results}

3.1. General Situation. The youth observation group consisted of 223 cases, 170 (76.2\%) male and 53 (23.8\%) female. The youth control group consisted of 158 cases, 111 (70.3\%) male and 47 (29.7\%) female.

3.2. Risk Factors Analysis for Observation Group and Youth Group. Univariate analysis showed that the following were risk factors for youth IS: hypertension, hyperlipidaemia, diabetes, family history of stroke, high homocysteine, quality of sleep, alcohol consumption, and smoking history. They all had statistical differences between observation and youth control group $(P<0.05)$. The proportion of cases with poor sleep quality in patients and in healthy young people was 47.1 and $29.1 \%$, respectively, and the difference between the two groups was statistically significant $(P<0.001)$ (Table 1$)$. However it is difficult to say how much the poor sleep quality was different between two groups. Seven risk factors (hypertension, hyperlipidaemia, family history of stroke, high homocysteine, quality of sleep, alcohol consumption, and smoking history) were screened by univariate analysis. They were made as independent variables and youth ischemic stroke was made as dependent variables. A multivariate logistic regression was conducted to identify statistically significant variables associated with youth IS. Arranged in
TABLE 2: Multivariate logistic regression analysis of risk factors for youth ischemic stroke. By multivariate logistic regression analysis, relative risk factors for youth IS, from highest to lowest, were hypertension, hyperlipidaemia, smoking history, high homocysteine, and poor sleep quality.

\begin{tabular}{lccc}
\hline & OR & OR (95\% CI) & $P$ \\
\hline Hypertension & 3.153 & $1.874-5.304$ & 0.000 \\
Hyperlipidaemia & 2.205 & $1.298-3.745$ & 0.003 \\
Smoking history & 2.091 & $1.257-3.479$ & 0.004 \\
High homocysteine & 2.051 & $1.230-3.421$ & 0.006 \\
Sleep quality & 1.811 & $1.116-2.939$ & 0.016 \\
Family history of stroke & 1.757 & $1.029-3.002$ & 0.039 \\
Drinking history & 1.718 & $1.074-2.749$ & 0.024 \\
\hline
\end{tabular}

order of significance, the five relative risk factors for youth IS, from highest to lowest, were hypertension, hyperlipidaemia, smoking, high homocysteine, and poor sleep quality (Table 2).

3.3. Short-Term Prognosis for Youth IS. Univariate analysis showed that the prognosis of young stroke patients included the following factors: NIHSS score, quality of sleep, and high homocysteine $(P<0.05)$ (Table 3$)$. The aforementioned significant variables were put into logistic regression models. Poor sleep quality was associated with the three-month MRS scores of youth IS patients (OR: 1.829; 95\% CI: 1.014-3.301). NIHSS score and high hyperlipidaemia were also associated with the three-month MRS scores of youth IS patients (Table 4).

\section{Discussion}

In recent years, the incidence of ischemic stroke in young people has increased and has received growing attention. The aetiology of IS is very complex; researchers have continued to investigate various risk factors for ischemic stroke with the aim of preventing youth IS by controlling its risk factors. Recently, the importance of identifying risk factors for 
TABLE 3: Univariate analysis of short-term prognostic factors in youth IS patients. By univariate analysis, NIHSS score, poor sleep quality, and high homocysteine were statistically different between living self-care and living dependent patients $(P<0.05)$.

\begin{tabular}{|c|c|c|c|c|}
\hline Factors & Daily living self-care $(\mathrm{MRS}<3)(n=150)$ & Living dependence $(\mathrm{MRS} \geq 3)(n=73)$ & Statistic & $P$ \\
\hline Gender (male) & $115(76.7)$ & $55(75.3)$ & 0.048 & 0.827 \\
\hline Age & $38.13 \pm 6.25$ & $38.55 \pm 6.58$ & 0.048 & 0.827 \\
\hline NIHSS & $5.5(3,8)$ & $9(6,15)$ & 5.113 & 0.000 \\
\hline TC & $1.72 \pm 1.11$ & $1.44 \pm 0.93$ & 3.580 & 0.06 \\
\hline TG & $4.53 \pm 1.38$ & $4.45 \pm 1.20$ & 1.247 & 0.265 \\
\hline HDL & $1.25 \pm 0.49$ & $1.28 \pm 0.34$ & 1.287 & 0.258 \\
\hline LDL & $2.96 \pm 1.04$ & $3.04 \pm 1.03$ & 0.322 & 0.571 \\
\hline Admission fasting glucose & $5.08 \pm 2.54$ & $5.20 \pm 2.27$ & 0.878 & 0.350 \\
\hline Sleep quality (poor) & $62(41.3)$ & $43(58.9)$ & 6.085 & 0.014 \\
\hline High homocysteine & $50(34.5)$ & $42(57.5)$ & 10.578 & 0.001 \\
\hline
\end{tabular}

TABLE 4: Logistic regression analysis of prognostic factors in youth IS patients. By multivariate logistic regression analysis, association with the three-month MRS scores, from highest to lowest, were NIHSS score, high homocysteine and poor sleep quality.

\begin{tabular}{lccc}
\hline Variable & OR & OR (95\% CI) & $P$ \\
\hline NIHSS & 2.846 & $1.475-5.491$ & 0.002 \\
Sleep quality (poor) & 1.829 & $1.014-3.301$ & 0.045 \\
High homocysteine & 2.232 & $1.226-4.062$ & 0.009 \\
\hline
\end{tabular}

ischemic stroke prevention has gained widespread concern. Youth IS risk factors include modifiable and nonmodifiable factors. Modifiable risk factors include smoking, drinking, hypertension, diabetes, hyperlipidaemia, high homocysteine, sleep quality, and eating habits. Nonmodifiable risk factors include age, gender, race, region of residence, and genetic factors. Studies conducted both in and out of China have shown that hypertension, hyperlipidaemia, and smoking are the most common risk factors for youth IS. Research shows that, out of China, the predominant risk factors were smoking and hypertension. In China, the predominant risk factors were high blood pressure and hyperlipidaemia [17].

Sleep is important to maintain the physiological processes of human life and for the protection of cortical inhibition. Adequate sleep is also essential to protecting brain cell energy metabolism. Studies have shown that sleep disorders are closely related to stroke risk factors such as diabetes, hypertension, and obesity. Research on the relationship between sleep quality and stroke has attracted widespread interest [18].

4.1. Relationship between Youth IS Onset and Sleep Quality. After comparing young patients and young healthy people by gender, age, condition, and rehabilitation of many risk factors, this study showed that male sex, hypertension, hyperlipidaemia, high blood homocysteine, diabetes, family history, poor quality of sleep, smoking, and alcoholism were associated with the incidence of stroke in young people. In this study, univariate analysis showed a statistically significant difference in sleep quality, with a higher proportion of poor sleep quality in youth IS patients (47.1\%) compared with healthy individuals (29.1\%). Results of the multivariate logistic regression analysis showed that poor sleep quality was the fifth strongest predictor of youth IS, indicating that this factor cannot be ignored and plays an important role in the pathogenesis of youth IS. The following reasons for poor sleep quality and insomnia were reported: pressure from work and life, poor self-control, addiction to the Internet, addiction to television, drinking, playing cards, excessive entertainment, modern nightlife, literacy, living alone, negative emotion, and mental health diagnosis. This study found that poor sleep quality is associated with the incidence of youth IS and is a powerful factor in youth IS onset. Attention to sleep quality provides guidance for youth IS prevention and has important clinical and social significance.

4.2. Relationship between Sleep Quality and Youth IS ShortTerm Prognosis. For youth IS, neurological impairment and restoration of life skills are important prognostic indicators. There are many evaluation studies of stroke scales used to measure the severity of stroke, and, of these, the NIHSS has demonstrated the highest degree of validity and reliability and is the most widely used. In one study, the NIHSS score at three months was strongly associated with the degree of independence of patients [19]. In this study, the NIHSS score was an independent predictor of poor youth IS prognosis (OR: 2.846; 95\% CI: 1.475-5.491). Epidemiological studies have shown that 15 to $35 \%$ of adults have sleep problems [20]. The level of sleep quality may differ across countries and regions due to various ethnic, geographical, economic, and cultural factors. Differences in the results of studies also have a strong relationship with the sampling method, survey group, and diagnostic criteria. This study investigated sleep quality as a prognostic variable and analysed its influence on youth IS prognosis. The results showed that poor sleep quality is associated with poor prognosis in youth IS patients (OR: 1.829 ; 95\% CI: 1.014-3.301), suggesting that good quality sleep may improve the prognosis of youth IS patients.

This study showed that there was a greater proportion of young male IS patients compared with female patients. This finding is consistent with the existing Chinese literature. Both similarities and differences between Chinese and other areal research have been demonstrated in risk factors associated with youth IS shown in the areas of youth IS pathogenesis. 
Logistic multivariate analysis showed that sleep quality was the fifth strongest predictor of youth IS. Thus, poor sleep quality is a significant contributor to the pathogenesis of youth IS. Youth IS is influenced by many factors. Risk factors and results identified in Chinese and other areal research differ due to different regions, climates, foods, cultures, and living habits.

Youth IS prevention in patients with poor compliance, the resultant impact on families and society, and the increasing IS incidence are all worthy of concern. Deep discussion of the risk factors related to youth IS, prognostic risk factors, and preventive measures, in addition to actively improving youth IS patient compliance, is our imperative duty and mission as medical workers. Clinical work should promote youth IS patients to develop good habits, provide universal health education about stroke, and understand the risk factors, especially the dangers of poor sleep quality. Appropriate primary and secondary prevention can effectively reduce the incidence of stroke in young people.

The limitations of this study included presence of an insufficient sample size and lack of basic research on sleep quality and youth IS onset. From the perspective of evidencebased medicine, further scientific evaluation of the relationship between sleep quality and youth IS incidence and prognosis using larger study samples and a multicentre randomized experimental design is needed. Whether poor sleep quality is an independent causal risk factor of youth IS incidence and prognosis requires further discussion and study. The positive correlation found between the risk factors and incidence of youth IS must be confirmed by further studies.

\section{Disclosure}

Shunqing Zhang and Cheng Chang are the co-first authors.

\section{Conflict of Interests}

The authors declare that there is no conflict of interests regarding the publication of this paper.

\section{Authors' Contribution}

Shunqing Zhang and Cheng Chang have contributed equally to this work.

\section{References}

[1] N. Yang, B. Zhang, and C. Gao, "The baseline NIHSS score in female and male patients and short-time outcome: a study in young ischemic stroke," Journal of Thrombosis and Thrombolysis, vol. 37, no. 4, pp. 565-570, 2014.

[2] J. Putaala, A. J. Metso, T. M. Metso et al., "Analysis of 1008 consecutive patients aged 15 to 49 with first-ever ischemic stroke the Helsinki young stroke registry," Stroke, vol. 40, no. 4, pp. 11951203, 2009.

[3] A. Bersano, L. Borellini, C. Motto et al., "Molecular basis of young ischemic stroke," Current Medicinal Chemistry, vol. 20, no. 31, pp. 3818-3839, 2013.
[4] S. S. Yang and J. P. Jia, "Differences in risk factors between anterior and posterior circulation affecting young ischemic stroke onset and prognosis," Zhonghua Yi Xue Za Zhi, vol. 93, no. 5, pp. 348-351, 2013.

[5] J. Putaala, E. Haapaniemi, M. Kurkinen, O. Salonen, M. Kaste, and T. Tatlisumak, "Silent brain infarcts, leukoaraiosis, and long-term prognosis in young ischemic stroke patients," Neurology, vol. 76, no. 20, pp. 1742-1749, 2011.

[6] F. Y. Khan, "Risk factors of young ischemic stroke in Qatar," Clinical Neurology and Neurosurgery, vol. 109, no. 9, pp. 770773, 2007.

[7] B. Gao, E. Cam, H. Jaeger, C. Zunzunegui, J. Sarnthein, and C. L. Bassetti, "Sleep disruption aggravates focal cerebral ischemia in the rat," Sleep, vol. 33, no. 7, pp. 879-887, 2010.

[8] P. Rocha, M. Barroso, A. Dantas, L. P. Melo, and T. F. Campos, "Predictive factors of subjective sleep quality and insomnia complaint in patients with stroke: implications for clinical practice," Anais da Academia Brasileira de Ciências, vol. 85, no. 3, pp. 1197-1206, 2013.

[9] M. Suh, S. Choi-Kwon, and J. S. Kim, "Sleep disturbances after cerebral infarction: role of depression and fatigue," Journal of Stroke and Cerebrovascular Diseases, 2014.

[10] H. P. Adams Jr., G. Del Zoppo, M. J. Alberts et al., "Guidelines for the early management of adults with ischemic stroke: a guideline from the American Heart Association," Stroke, vol. 38, no. 5, pp. 1655-1711, 2007.

[11] M. E. Hall, J. M. do Carmo, A. A. da Silva et al., "Obesity, hypertension, and chronic kidney disease," International Journal of Nephrology and Renovascular Disease, vol. 18, no. 7, pp. 75-88, 2014.

[12] S. A. Saha and R. R. Arora, "Hyperlipidaemia and cardiovascular disease: do fibrates have a role?" Current Opinion in Lipidology, vol. 22, no. 4, pp. 270-276, 2011.

[13] M. M. Castañon, A. M. Lauricella, L. Kordich, and I. Quintana, "Plasma homocysteine cutoff values for venous thrombosis," Clinical Chemistry and Laboratory Medicine, vol. 45, no. 2, pp. 232-236, 2007.

[14] P. Z. Zimmet, D. J. Magliano, W. H. Herman, and J. E. Shaw, "Diabetes: a 21st century challenge," The Lancet Diabetes and Endocrinology, vol. 2, no. 1, pp. 56-64, 2014.

[15] A. M. Hashmi, I. S. Khawaja, Z. Butt et al., “The Pittsburgh sleep quality index: validation of the Urdu translation," Journal of the College of Physicians and Surgeons-Pakistan, vol. 24, no. 2, pp. 123-126, 2014.

[16] N. Weisscher, M. Vermeulen, Y. B. Roos, and R. J. De Haan, "What should be defined as good outcome in stroke trials; a modified Rankin score of 0-1 or 0-2?" Journal of Neurology, vol. 255, no. 6, pp. 867-874, 2008.

[17] A. Pezzini, M. Grassi, E. del Zotto et al., "Synergistic effect of apolipoprotein E polymorphisms and cigarette smoking on risk of ischemic stroke in young adults," Stroke, vol. 35, no. 2, pp. 438-442, 2004.

[18] A. Ferre, M. Ribó, D. Rodríguez-Luna et al., "Strokes and their relationship with sleep and sleep disorders," Neurología, vol. 28, no. 2, pp. 103-118, 2013.

[19] K. C. Johnston and D. P. Wagner, "Relationship between 3month National Institutesof Health Stroke Scale score and 
dependence in ischemic stroke patients," Neuroepidemiology, vol. 27, no. 2, pp. 96-100, 2006.

[20] D. J. Buysse, C. F. Reynolds III, T. H. Monk, S. R. Berman, and D. J. Kupfer, "The Pittsburgh sleep quality index: a new instrument for psychiatric practice and research," Psychiatry Research, vol. 28, no. 2, pp. 193-213, 1989. 


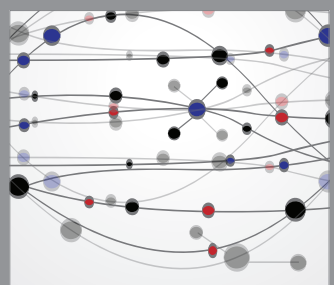

The Scientific World Journal
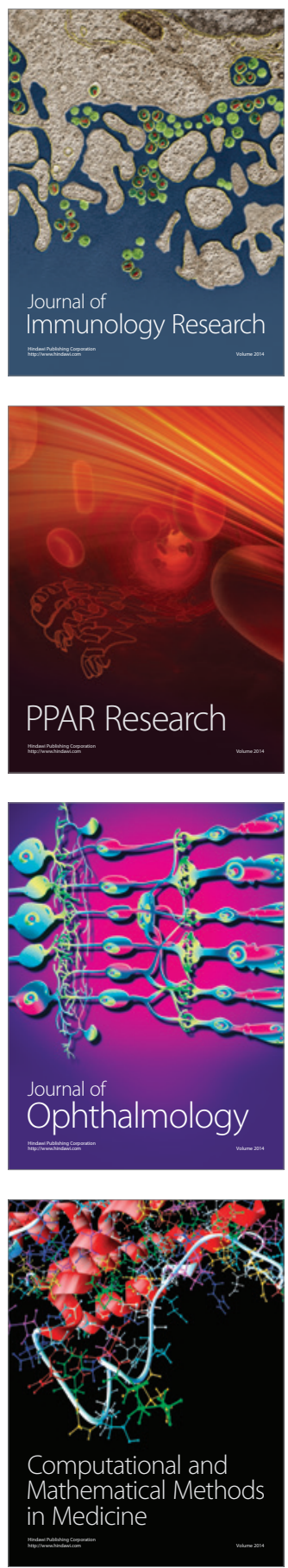

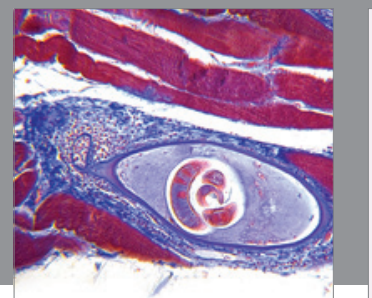

Gastroenterology

Research and Practice
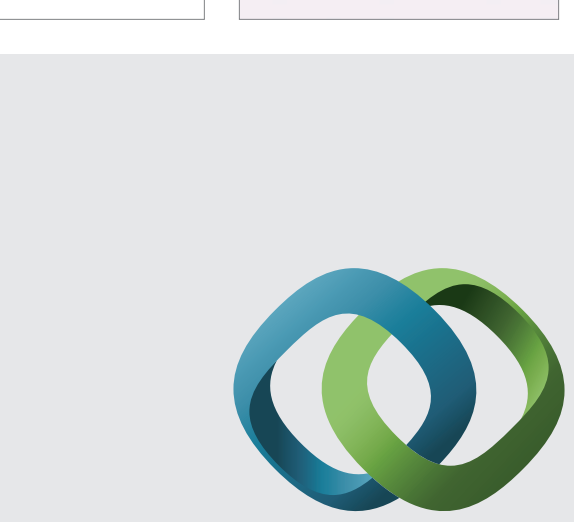

\section{Hindawi}

Submit your manuscripts at

http://www.hindawi.com
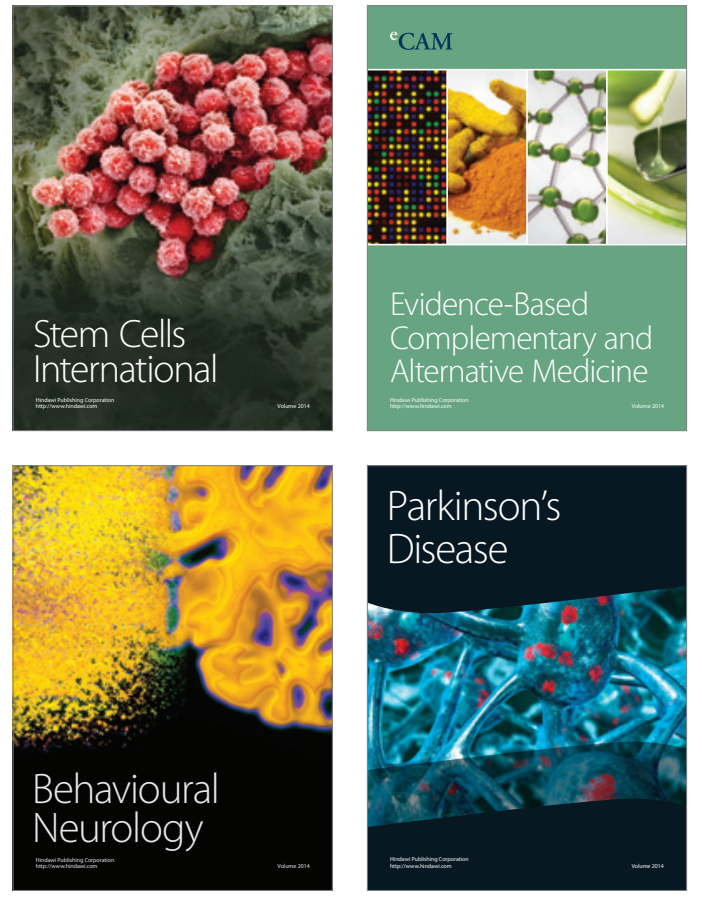
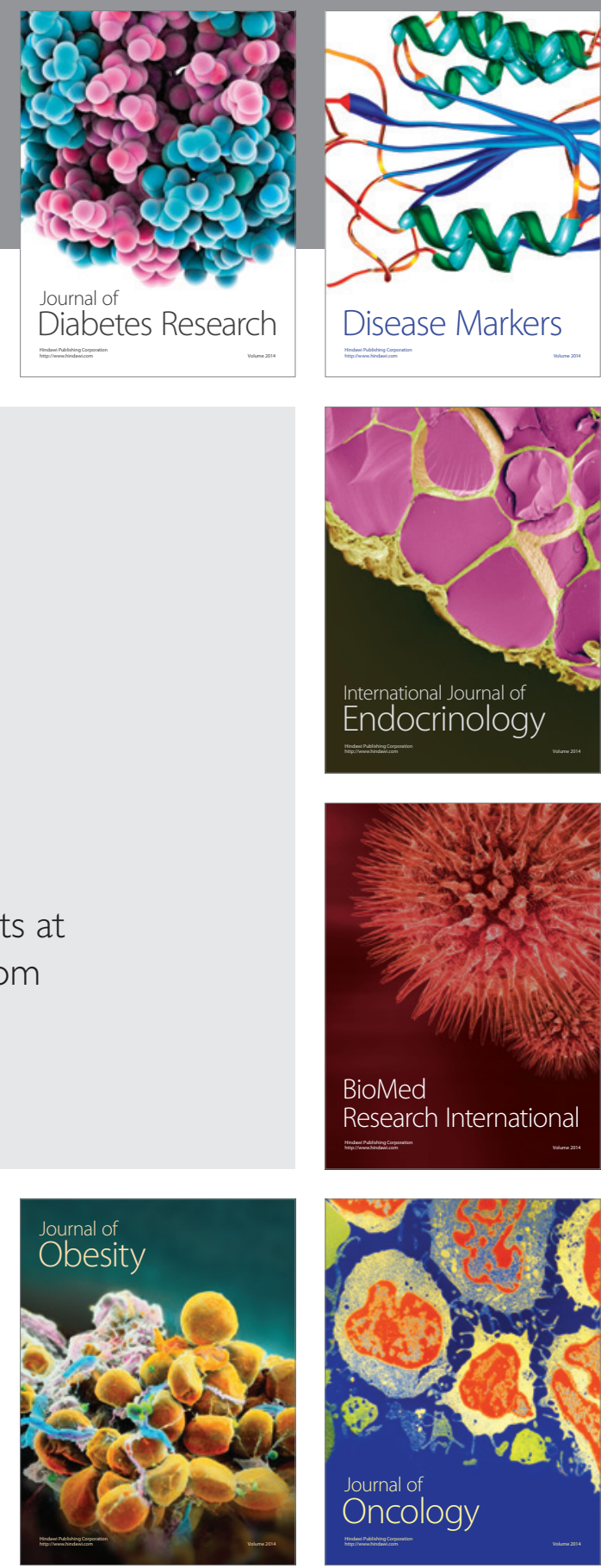

Disease Markers
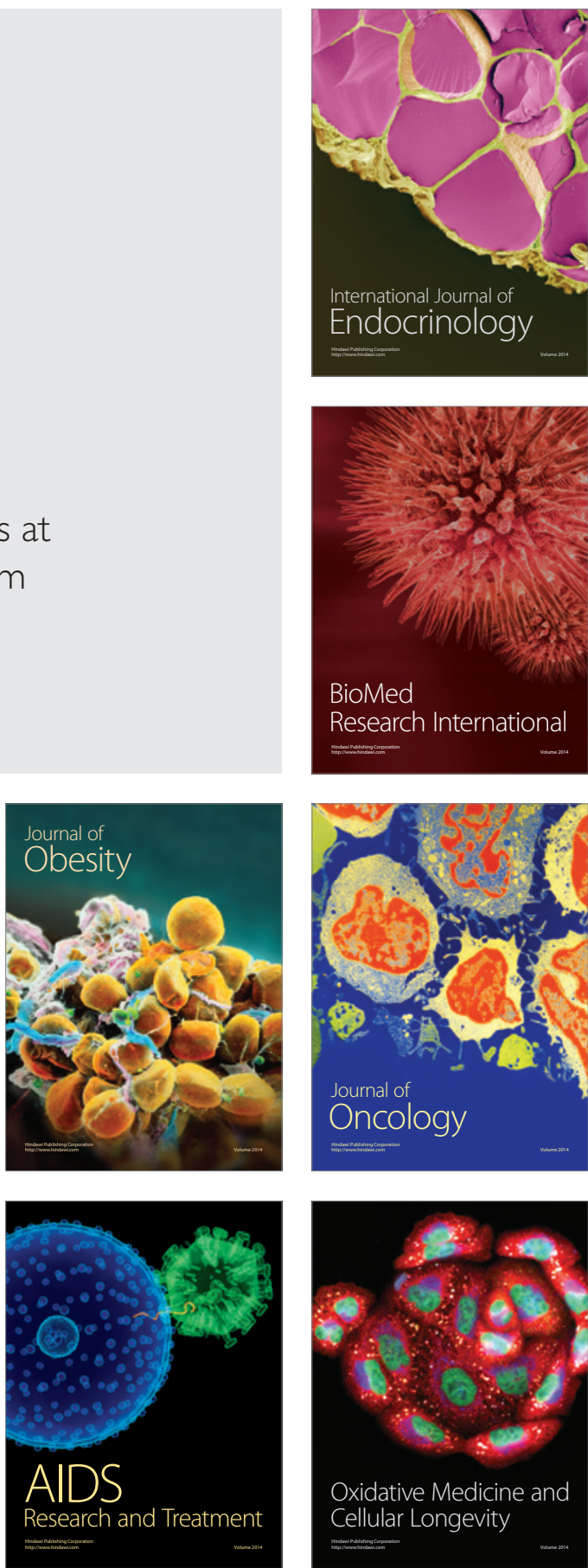